diarrhoea and/or vomiting are seen by an assessment nurse and a junior doctor or general practitioner, spending over two and half hours in the emergency department.

Assessment of problem and analysis of its causes We quantified the scale of the problem by undertaking a retrospective audit of the care children received when they presented with gastroenteritis. Ninety percent of children who presented had a very mild illness that could have been managed safely at home. We supplemented the quantitative data with informal conversations with colleagues. There was a widespread view that many parents presented to hospital due to anxiety and/or uncertainty about how to manage their child's illness at home.

We extracted data from the electronic medical records of 580 children presenting with diarrhoea and/or vomiting. The concordance between a final diagnosis of viral gastroenteritis and the parents reporting diarrhoea or vomiting at reception was $97 \%$ and $90 \%$ respectively. Such a high concordance between symptoms and diagnosis reassures us that the assessment nurse could accurately diagnose gastroenteritis. We calculated the relative risk of serious illness depending on the presence of 16 easy to illicit clinical symptoms and signs. Based on these findings we have designed a pathway that the triage nurse will use to discharge patients. We have retrospectively tested the efficacy and safety of this new pathway. Our research suggests that half of these patients could be safely discharged by the assessment nurses using our simple protocol.

Intervention We designed a criteria based discharge pathway, allowing nurses to safely discharge patients from triage without waiting to see a doctor. The discharge pathway is supported by a multilingual patient and parent education video that can be viewed in the department and on the trust website.

Strategy for change After designing the discharge pathway we presented it at departmental meetings and teaching sessions in order to get feedback. Once the pathway was approved we identified'champions' who have supported its implementation and have helped to train staff.

Measurement of improvement We designed a dashboard providing a visual display of the four key performance indicators: number of cases of diarrhoea and vomiting, percentage of cases discharged on the pathway, mean duration of stay in the emergency department and reattendance rate.

Effects of changes 42 children have been safely discharged on the new pathway. Anecdotal feedback has been very positive. It is too early to measure the impact of the new pathway on mean duration of stay in the emergency department and reattendance rate.

Lessons learnt It takes time to embed new pathways into routine care and engage stakeholders. It has been very important to build a team of people to champion the new pathway.

Message for others This has been a really exciting project and was enthusiastically received by staff. It represents an innovative way of managing simple paediatric presentations and has the potential to deliver a better service to children and their parents and, we hope, a longer term effect as we are educating parents and giving them a resource they can refer back to.

Please declare any conflicts of interest

The video was funded by the Barts and The London Charity.

\section{G592 EMPOWERING PARENTS TO MANAGE EVERYDAY CHILDHOOD ILLNESS AT HOME}

R Justice. Health Education Wessex, Health Education England, Winchester, UK

10.1136/archdischild-2015-308599.541
Context The project was part of a Quality Improvement Fellowship. It worked directly with the local Sure Start children centres. It worked alongside a local strategy to provide paediatric patients with the best care in the most appropriate setting.

Problem The aim was to enable children centre staff to empower parents and provide reassurance on managing everyday childhood illnesses at home.

Assessment of problem and analysis of its causes A Pre-project questionnaire, completed by 85 parents, demonstrated that most take their children to the emergency department seeking reassurance.

The project worked with 2 of 4 local children's 'Sure Start' centres to provide parents with key points to manage $3 \mathrm{com}$ monly seen childhood illnesses at home. The focus was on empowering parents and reassuring them to trust their own instincts.

Intervention A facilitator guide and parent information leaflet was produced using information already in circulation.

Six childhood illness sessions were run, by the author, during parent and children groups in Sure Start. This was both a demonstration to Sure Start facilitators and gained parent feedback.

At the end of each session verbal feedback from facilitators and parents was reviewed and material revised (PDSA cycles). The final version incorporated basic 'games' such as true/ false flash cards and question and answer flash cards.

Parents stated they found the information 'useful', 'reassuring' or 'helpful'. Facilitators found the material 'easy to use' and described the sessions as 'engaging'. They would be able to run a session themselves.

A working group was set up to embed the sessions into the regular groups.

Strategy for change The material was introduced at the Sure Start Team Lead meetings and parent groups over a 4 month period. The facilitators had opportunity to review the material and observe sessions. They were directly observed and supported when running their first session.

The CCG Lead for Sure Start was involved throughout and was key in helping to support the program.

A working group of experienced staff was created by the Sure Start Team Lead. The current pilot will run over 6 months

Measurement of improvement The material was trialled and reviewed by requesting verbal feedback from both parents and facilitators. The initial three sessions were well received by parents. Facilitators felt apprehensive about responding to medical questions. 7 out of 9 were not confident to run a session alone.

More structure and guidance was added and the information was put into a 'game 'format'. 3 revised sessions were run. After observing, 7 out of 7 (100\%) group facilitators were confident to run a session.

A further process measure was the numbers of regular sessions run by confident group facilitators within children centres. This is currently 3 a month in one of the local areas.

Effects of changes The parents exposed to the sessions gave positive feedback such as 'If I had known this before I wouldn't have worried' or 'It's good to have people coming into groups to talk to parents about things like that'.

The main problem was engaging the Sure Start centre staff to lead a session. They liked the material and the sessions but felt apprehensive about leading a session. It took longer than expected to engage them.

Lessons learnt I have learnt how important engagement is.

Next time I will set up a working group earlier to gain better engagement. 
Message for others My project has highlighted how important it is to involve patients in new initiatives.

For paediatric patients it is really important to understand why parents bring their children in as an emergency.

For change to happen it is important to engage and empower parents to make the right choices for their children.

\section{G593 TEAM POD: A QUALITY IMPROVEMENT SPRINT TO IMPROVE PATHOLOGY SERVICES}

${ }^{1}$ 1S Blackstock, ${ }^{11} \mathrm{R}$ Irvine, ${ }^{22} \mathrm{~F}$ Ejiofor, ${ }^{33} \mathrm{~S}$ Popa, ${ }^{44 \mathrm{~J}}$ Tarma, ${ }^{44} \mathrm{~A}$ Furetta, ${ }^{11} \mathrm{~F}$ Cleugh. ${ }^{1}$ Paediatric Department, St Marys Hospital, Imperial College Healthcare NHS Trust, London, UK; ${ }^{2}$ Quality and Logistics Manager, Pathology Business Services, St Marys Hospital, Imperial Hospital Healthcare NHS Trust, London, UK; ${ }^{3}$ Bioengineering, Imperial College London, London, UK; ${ }^{4}$ Service Design, Royal College of Art, London, UK

\subsection{6/archdischild-2015-308599.542}

Whilst working as trainees in a Paediatric department, it became apparent that there were significant delays in patient pathology results. This impacted on patient care, delaying management decisions and discharges, as well as wasting clinicians' time and hospital resources.

We brought this specific issue to a Quality Improvement Sprint; a 2 day hackathon, which served as a catalyst for change.

Our team of doctors, managers, design students and an engineer (who subsequently became known as Team Pod) performed an onsite analysis of the process from patient bedside to pathology laboratory. The wide range of skill sets and experiences within the team were invaluable for this process. We spent time on the wards, shadowing porters, interviewed staff and using the systems in place, gaining valuable insights into the multifaceted problem.

Many hindrances were highlighted. Equipment on the ward was poorly organised and sample volumes required for various investigations were unclear. Although the hospital had a pneumatic rapid delivery system (Pod System), which took minutes to deliver samples to the laboratory, it was rarely used by ward staff. Most samples were being transferred via a porter, which could take hours. A small portering team covers a vast number of clinical areas. Most pod stations had insufficient or no pods available, and there was a general notion amongst staff that the pod system was 'unreliable.'

Fascinatingly, we discovered that the porters were among the highest users of the pod system. They were gathering samples, then sending them to the laboratories using the pod station in their porters' lodge. Often the porters were even unable to access the laboratories after hours so could not hand-deliver. Furthermore, many of the pods were unlabeled, so laboratory staff did not know which wards to return them to, and therefore sent them to the highest users (A\&E or the Porters) perpetuating the cycle. We calculated that the overall inefficiencies in the blood sampling and transfer process was costing the trust an estimated $£ 1.7$ million per year (time spent finding equipment, waiting for porters, calling up laboratories, repeating tests, and delayed discharges).

Having better identified the nature of the inefficiencies, we formulated a problem statement, and proposed distinct initiatives. This included a re-organisation of equipment on the wards, which we instigated via the ward managers. A paediatric specific reference chart with accurate blood volumes and bottles for tests has been developed. We also approached security services and ensured that all porters had appropriate access to the laboratories during and out of hours. Most significantly, we presented our findings and a business case to the trust pathology

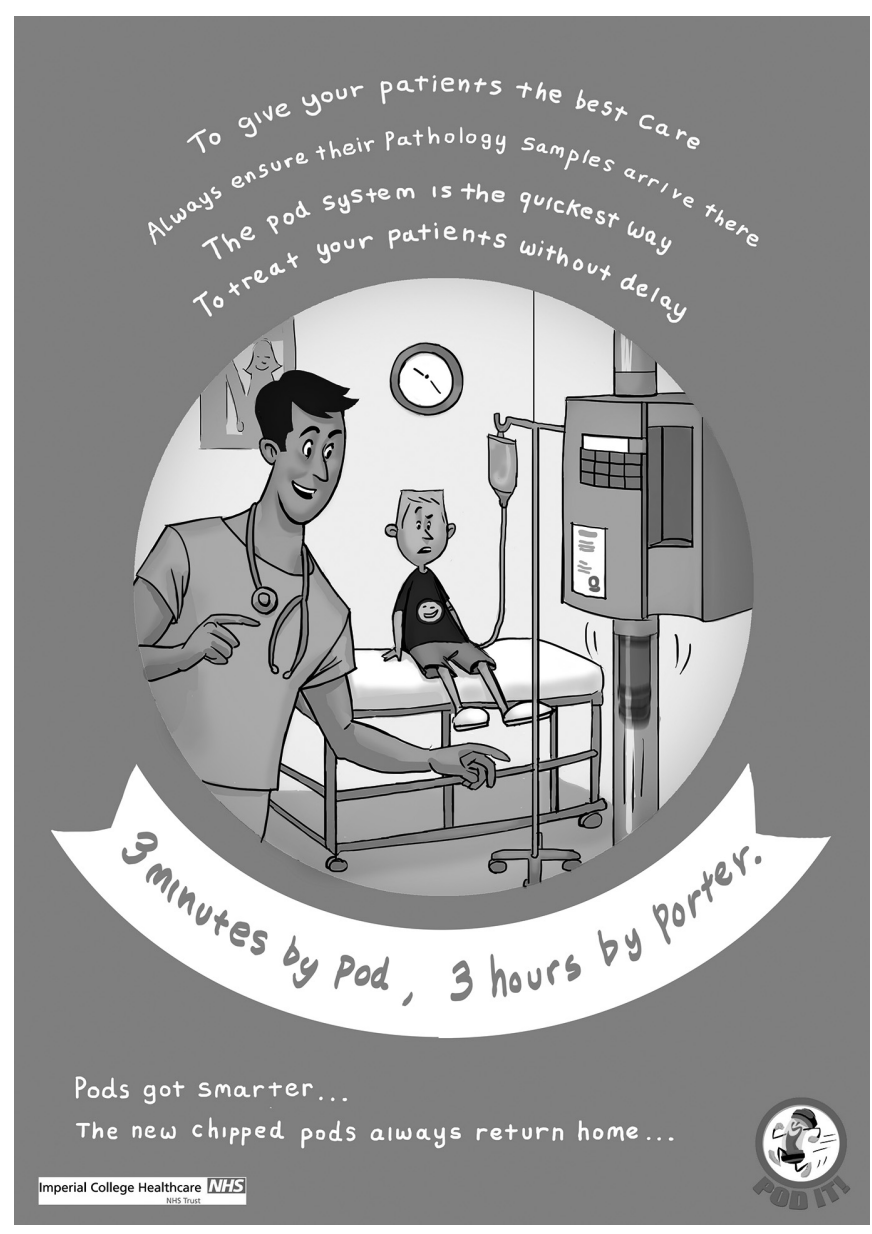

Abstract G593 Figure 1

lead, and a new pod system with trackable 'chipped' pods was approved and implemented. The chipped pods are programmed to always return to their designated station. We then launched a 'pod campaign' to raise awareness of the new system within the trust. The aim was to change the culture and engage staff to realise the benefits and time saved by using the new pod system.

This improvement project demonstrates how basic practices within a hospital can be improved if staff take ownership of the systems they use and engage in efforts to understand and improve processes, and change culture. It was hugely beneficial to have a team with varied experiences and perspectives, and to have dedicated time to focus on a particular issue. Following the two-day hackathon, continued significant efforts were required to see the changes delivered, but the team were motivated and enthused to make a difference. By running these events regularly within NHS trusts, it is possible to mobilise staff to initiate improvements for the care of their patients.

\section{G594 TACKLING HIGH MORTALITY IN A KENYAN DISTRICT HOSPITAL}

B Cahill. Global Links, Royal College of Paediatrics and Child Health, London, UK

\subsection{6/archdischild-2015-308599.543}

Context Completed in a Kenyan hospital paediatric Emergency Department. Clinical officers, Nurses and administration team involved. 